\title{
Noncompaction cardiomyopathy: A rare cardiomyopathy
}

\author{
Sachin Kumar Amruthlal Jain, Brijesh Patel, Timothy R Larsen, \\ Yousif Ismail, Shukri David
}

\begin{abstract}
Introduction: Noncompaction cardiomyopathy (NCCM) is a rare form of cardiomyopathy. The American Heart Association classified it as a genetic cardiomyopathy. The pathogenesis of this condition is attributed to the failure of trabecular myocardium to compact and underdeveloped microcoronary circulation. Case Report: We present a case of a 72-year-old African American female who presented with shortness of breath and leg swelling and was found to have severe systolic dysfunction. Stress test showed ischemia. Left heart catheterization which included left ventriculogram showed prominent trabeculations suggestive of noncompaction cardiomyopathy. Conclusion: This condition is often associated with other congenital cardiac defects and arrthymias. When it is present alone, it is called isolated noncompaction cardiomyopathy (INCCM). The treatment protocol for this cardiomyopathy is the same as other cardiomyopathies, and includes the management of arrhythmias, heart failure and potential risk of embolism.
\end{abstract}

Keywords: Noncompaction cardiomyopathy, Echocardiography, Heart failure, Congenital heart disease

Sachin Kumar Amruthlal Jain ${ }^{1}$, Brijesh Patel ${ }^{1}$, Timothy $\mathrm{R}$ Larsen $^{1}$, Yousif Ismail ${ }^{1}$, Shukri David ${ }^{1}$

Affiliations: ${ }^{1} \mathrm{MD}$, Providence Hospital and Medical Center, Southfield, MI.

Corresponding Author: Sachin Kumar Amruthlal Jain, MD, Department of Cardiology, Providence Hospital and Medical Centers, 16001 W. Nine Mile Road, Southfield, MI. 48075; Phone: 248849 3968; Fax: 248849 3151; E-mail: doctorsachin@gmail.com

Received: 15 October 2012

Accepted: 23 February 2013

Published: 01 September 2013
Jain SKA, Patel B, Larsen TR, Ismail Y, David S. Noncompaction cardiomyopathy: A rare cardiomyopathy. International Journal of Case Reports and Images 2013;4(9):467-470.

$* * * * * * * * *$

doi:10.5348/ijcri-2013-09-358-CR-2

\section{INTRODUCTION}

Noncompaction cardiomyopathy (NCCM) is a rare form of cardiomyopathy $[1,2]$. The American Heart Association classifies it as a genetic cardiomyopathy [1]. The pathogenesis of this condition is attributed to the failure of trabecular myocardium to compact and underdeveloped microcoronary circulation. This condition is often associated with other congenital cardiac defects and arrthymias. When it is present alone then it is called isolated noncompaction cardiomyopathy (INCCM) $[1,2]$. Currently, the treatment protocol for this cardiomyopathy is the same as other cardiomyopathies, and includes the management of arrhythmias, heart failure and potential risk of embolism [2].

\section{CASE REPORT}

Our patient, was a 72-year-old African-American female who came to the emergency room (ER) complaining of lower extremity edema and shortness of breath for one week. She gave a history of worsening dyspnea and gradual development of orthopnea. She denied paroxysmal nocturnal dyspnea, cough, chest pain, sputum production or palpitation. She was hospitalized for congestive heart failure and lower extremity edema in the past. Her past medical history was significant for severe congestive heart failure, hypertension and chronic obstructive pulmonary disease. She was a smoker. Her 
physical examination was significant for shortness of breath and bilateral lower extremity edema (1+). Her medication included aspirin, carvedilol, furosemide, hydralazine, simvastatin and tiotropium. Her electro cardiogram showed atrial flutter with 2:1 block, bilateral atrial enlargement, Q-waves in Lead II and III, poor R-wave progression and prolonged QT interval (499 $\mathrm{ms})$. In the hospital, patient was treated for symptoms of congestive heart failure, and underwent ablation procedure for atrial flutter. The patient was in normal sinus rhythm and with improved symptoms at the time of discharge.

Image findings and analysis: The patient underwent echocardiographic study to assess the left ventricular function and right chamber pressure measurements, because of her symptoms and the history of severe congestive heart failure. The study showed enlarged left ventricle with severe global systolic dysfunction and depressed ejection fraction of $20 \%$, grade I diastolic impairment, mildly enlarged left atrium and a trace of mitral and pulmonary regurgitation. In addition, it showed trabeculations in the left ventricle (Figure 1). The severe global systolic dysfunction warranted further evaluation of her heart. Therefore, she underwent lexiscan cardiolite nuclear stress test. The stress test showed large partially reversible inferior and inferolateral defect consistent with stress induced ischemia and also noted to have dilated right ventricle. To further evaluate the ischemia, two days later, she underwent cardiac catheterization/ left ventriculogram that showed ejection fraction of $20 \%$, severe global impairment in contractility and prominent left ventricular trabeculation suggesting NCCM (Figure 2), compared to a normal left ventriculogram from another patient (Figure 3).

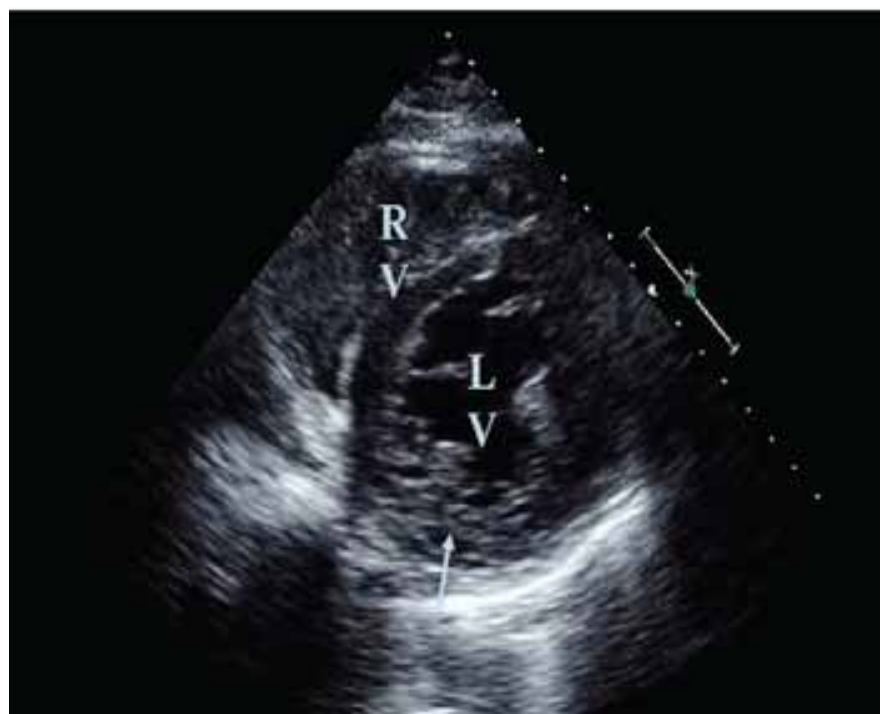

Figure 1: Transthoracic echocardiogram revealing prominent inferoapical trabeculations (arrow). LV-left ventricle, RV-right ventricle.

\section{DISCUSSION}

During the first five to eight weeks of embryonic life, spongy/trabecular myocardium starts to compact. These trabecular fibers that are separated by ventricular recesses link myocardium to the ventricular cavity. The noncompaction cardiomyopathy ensues when the myocardium fails to compact fully. The coronary circulation also develops during this period. When the fibers fail to compact they often have compromised coronary microcirculation which makes them more susceptible to ischemia and hypokinesia [2]. Many times, cases of noncompaction cardiomyopathy also have concurrent arrthymias and congenital cardiac defects. The image studies in our patient did not reveal any

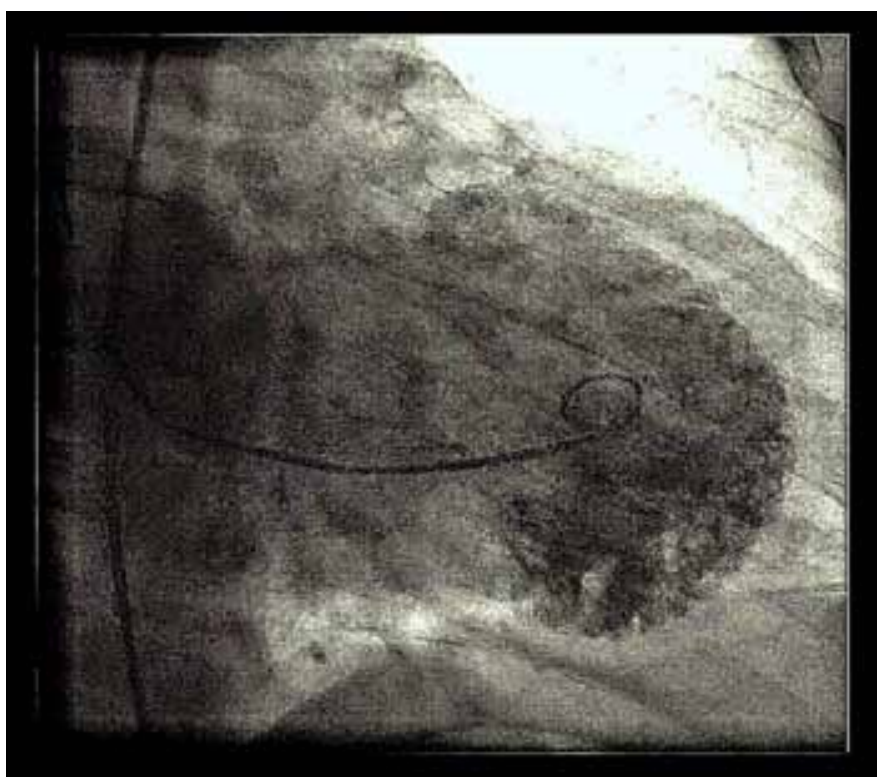

Figure 2: Left ventriculogram demonstrating prominent apical trabeculae (arrows). (L-left ventricular cavity).

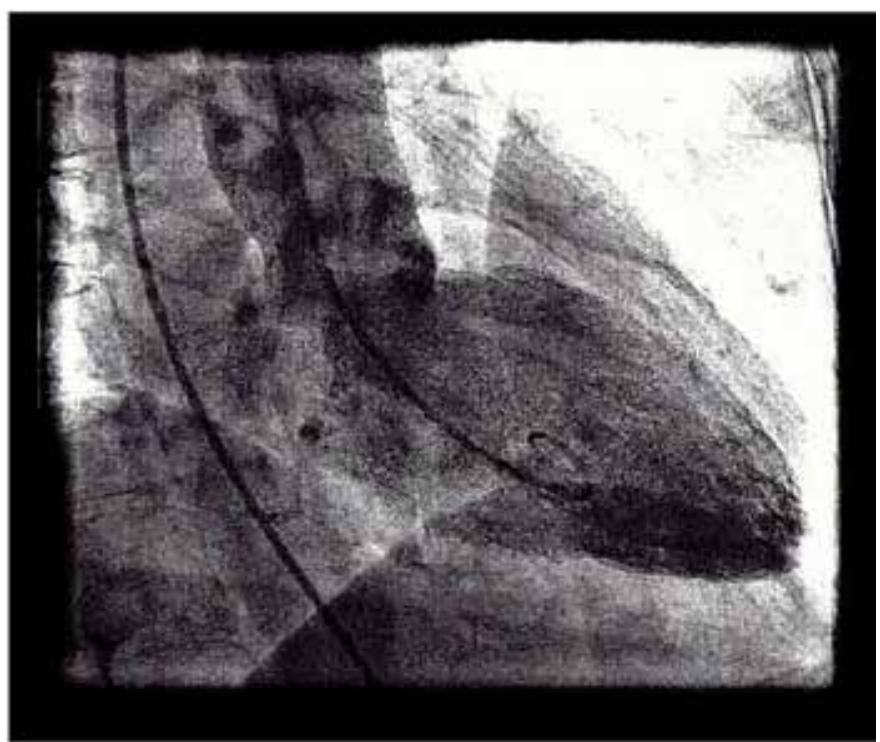

Figure 3: Left ventriculogram from another patient demonstrating normal left ventricular myocardium for comparison. 
congenital cardiac defect. Therefore, it is called isolated noncompaction cardiomyopathy.

In 2006, the American Heart Association included noncompaction cardiomyopathy as a genetic cardiomyopathy [1]. However, it may exist in both sporadic and familial forms. In case of familial form, it is associated with X-link inheritance linked to G4.5 gene (named Tafazzin) on Xq28 and a gene located on 11p15 [1]. The actual prevalence of noncompaction cardiomyopathy is unclear. The increasing number of case reports on this condition and various imaging options have helped to approximate the prevalence. Based on echocardiographic studies, $0.014-0.05 \%$ of the general population might be affected with this condition $[1,2]$. The affected patients may develop systolic or diastolic dysfunction, or both, at the same time [3]. Echocardiography has remained as the main modality for diagnosis of this condition [2]. However, other imaging modalities can offer more information about the structure and function of the heart. The differential diagnosis of isolated noncompaction cardiomyopathy include acquired, ischemic or mixed cardiomyopathy. Currently, it is managed as other cardiomyopathies, and includes appropriate treatment for heart failure, arrthymias and embolism [2]. Growing literature on noncompaction cardiomyopathy will enhance the understanding of pathogenesis, management and prognosis of this condition.

\section{CONCLUSION}

Noncompaction cardiomyopathy is a rare congenital heart condition that presents later in life. Patients with this condition present with heart failure, arrhythmias and/or embolism. In conclusion, patients with noncompaction cardiomyopathy are treated as other heart failure patients.

\section{Author Contributions}

Sachin Kumar Amruthal Jain - Conception and design, Acquisition of data, Analysis and interpretation of data, Critical revision of the article, Final approval of the version to be published

Brijesh Patel - Conception and design, Acquisition of data, Analysis and interpretation of data, Drafting the article, Critical revision of the article, Final approval of the version to be published

Timothy Larsen - Acquisition of data, Analysis and interpretations, Critical revision of the article, Final approval of the version to be published

Yousif Ismail - Acquisition of data, Analysis and interpretations, Critical revision of the article, Final approval of the version to be published

Shukri David - Acquisition of data, Analysis and interpretations, Critical revision of the article, Final approval of the version to be published

\section{Guarantor}

The corresponding author is the guarantor of submission.

\section{Conflict of Interest}

Authors declare no conflict of interest.

\section{Copyright}

(C) Sachin Kumar Amruthlal Jain et al. 2013; This article is distributed under the terms of Creative Commons attribution 3.0 License which permits unrestricted use, distribution and reproduction in any means provided the original authors and original publisher are properly credited. (Please see www.ijcasereportsandimages.com/ copyright-policy.php for more information.)

\section{REFERENCES}

1. Espinola-Zavaleta N, Soto ME, Castellanos LM, Játiva-Chávez S, Keirns C. Non-compacted cardiomyopathy: Clinical-echocardiographic study. Cardiovasc Ultrasound 2006;4:35.

2. Weiford BC, Subbarao VD, Mulhern KM. Noncompaction of the ventricular myocardium. Circulation 2004;109(24):2965-71.

3. Luong MW, Mettini S, Delgado D. Noncompaction Cardiomyopathy. UTMJ 2010;87:144-7. 
Access PDF of article on other devices

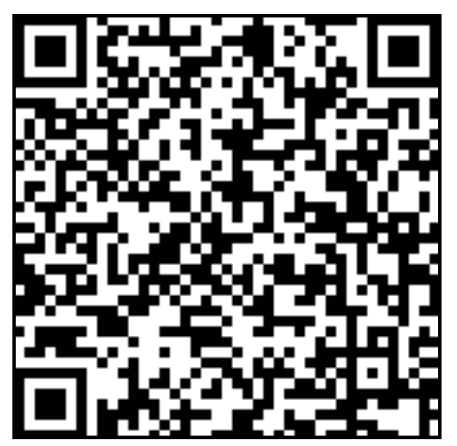

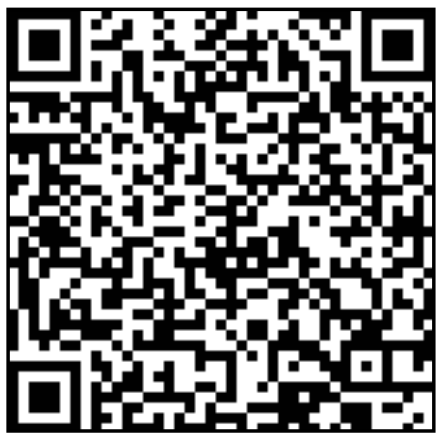

